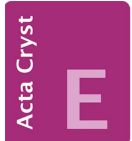
COMMUNICATIONS

ISSN 2056-9890

\title{
Crystal structure of 1,3-bis\{[4-(acetyl- sulfanyl)phenyl]ethynyl\}azulene
}

\section{Sebastian Förster, Wilhelm Seichter and Edwin Weber*}

Institut für Organische Chemie, TU Bergakademie Freiberg, Leipziger Strasse, 29, D09596 Freiberg/Sachsen, Germany. *Correspondence e-mail:

edwin.weber@chemie.tu-freiberg.de

Received 19 November 2015; accepted 7 January 2016

Edited by W. T. A. Harrison, University of Aberdeen, Scotland

In the title compound, $\mathrm{C}_{30} \mathrm{H}_{20} \mathrm{O}_{2} \mathrm{~S}_{2}$, the dihedral angles between the central azulene ring system (r.m.s. deviation $=$ $0.039 \AA$ ) and the pendant benzene rings are $28.96(7)$ and $55.15(7)^{\circ}$. The dihedral angles between the benzene rings and their attached acetylsulfanyl groups are $59.60(10)$ and $84.79(10)^{\circ}$. The expected $\pi-\pi$ stacking interactions are not observed in the crystal structure; instead, the packing features $\mathrm{C}-\mathrm{H} \cdots \mathrm{O}$ hydrogen bonds, which link the molecules into $C(12)$ [010] chains, which are supported by weak $\mathrm{C}-\mathrm{H} \cdots \pi$ contacts.

Keywords: crystal structure; azulene; 1,3-disubstitution; $\mathrm{C}-\mathrm{H}$... O hydrogen bond; $\mathrm{C}-\mathrm{H} \cdots \pi$ interaction.

CCDC reference: 1445850

\section{Related literature}

For background to this work, see: Wang et al. (2009); Puodziukynaite et al. (2014); Xia et al. (2014). For the synthesis and related structures, see: Förster et al. (2012, 2014).

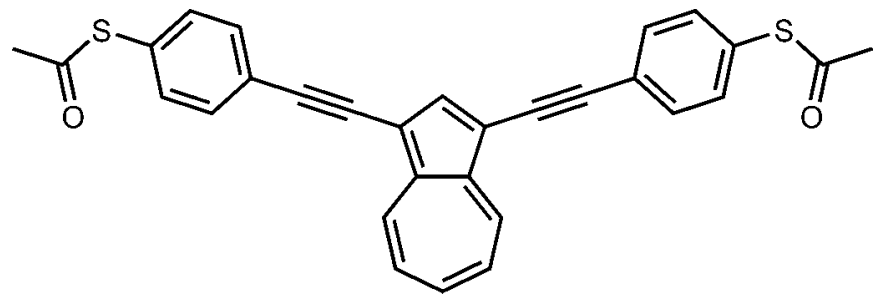

\section{Experimental}

2.1. Crystal data

$\mathrm{C}_{30} \mathrm{H}_{20} \mathrm{O}_{2} \mathrm{~S}_{2}$

$M_{r}=476.58$

Monoclinic, $P 2_{1} / n$

$$
\begin{aligned}
& a=13.7674(3) \AA \\
& b=8.9849(2) \AA \\
& c=19.7586(4) \AA
\end{aligned}
$$

$\beta=104.022(1)^{\circ}$

$V=2371.28(9) \AA^{3}$

$Z=4$

Mo $K \alpha$ radiation

$\mu=0.25 \mathrm{~mm}^{-1}$

$T=100 \mathrm{~K}$

$0.24 \times 0.23 \times 0.15 \mathrm{~mm}$

\subsection{Data collection}

Bruker Kappa APEX CCD diffractometer

Absorption correction: multi-scan (SADABS; Bruker, 2008)

$T_{\min }=0.942, T_{\max }=0.963$

\subsection{Refinement}

$R\left[F^{2}>2 \sigma\left(F^{2}\right)\right]=0.046$

$w R\left(F^{2}\right)=0.130$

$S=1.03$

5898 reflections

309 parameters

$\mathrm{H}$-atom parameters constrained

$\Delta \rho_{\max }=0.44{\mathrm{e} \AA^{-3}}^{-3}$

$\Delta \rho_{\min }=-0.27$ e $\AA^{-3}$

Table 1

Hydrogen-bond geometry $\left(\AA,^{\circ}\right)$.

$C g 1$ is the mid-point of the $\mathrm{C} 11-\mathrm{C} 12$ bond and $\mathrm{Cg} 2$ is the centroid of the $\mathrm{C} 1-$ C4/C10 ring.

\begin{tabular}{lllll}
\hline$D-\mathrm{H} \cdots A$ & $D-\mathrm{H}$ & $\mathrm{H} \cdots A$ & $D \cdots A$ & $D-\mathrm{H} \cdots A$ \\
\hline $\mathrm{C} 2-\mathrm{H} 2 \cdots \mathrm{O} 1^{\mathrm{i}}$ & 0.95 & 2.40 & $3.285(3)$ & 155 \\
$\mathrm{C} 17-\mathrm{H} 1 \cdots C g 1$ & 0.95 & 2.69 & $3.612(3)$ & 165 \\
$\mathrm{C} 20-\mathrm{H} 20 A \cdots C g 2^{\mathrm{ii}}$ & 0.98 & 2.89 & $3.835(2)$ & 162 \\
\hline
\end{tabular}

Symmetry code: (i) $-x+\frac{5}{2}, y-\frac{1}{2},-z+\frac{3}{2}$; (ii) $x+\frac{1}{2},-y+\frac{1}{2}, z-\frac{1}{2}$.

Data collection: APEX2 (Bruker AXS); cell refinement: SAINT (Sheldrick, 2008); data reduction: SAINT; program(s) used to solve structure: SHELXS97 (Sheldrick, 2008); program(s) used to refine structure: SHELXL2015 (Sheldrick, 2015); molecular graphics: ORTEP-3 for Windows (Farrugia, 2012); software used to prepare material for publication: SHELXTL (Sheldrick, 2008).

\section{Acknowledgements}

This work has been performed within the 'Cluster of Excellence Structure Design of Novel High-Performance Materials via Atomic Design and Defect Engineering' (ADDE), which was supported financially by the European Union (European Regional Development Fund) and by the Ministry of Science and Art of Saxony (SMWK).

Supporting information for this paper is available from the IUCr electronic archives (Reference: HB7546).

\section{References}

Bruker (2008). APEX2 and SAINT. Bruker AXS Inc., Madison, Wisconsin, USA.

Farrugia, L. J. (2012). J. Appl. Cryst. 45, 849-854.

Förster, S., Hahn, T., Loose, C., Röder, C., Liebing, S., Seichter, W., Eissmann, F., Kortus, J. \& Weber, E. (2012). J. Phys. Org. Chem. 25, 856-863.

Förster, S., Seichter, W., Kuhnert, R. \& Weber, E. (2014). J. Mol. Struct. 1075, 63-70.

Puodziukynaite, E., Wang, H.-W., Lawrence, J., Wise, A., Russell, T. P., Barnes,

M. D. \& Emrick, T. (2014). J. Am. Chem. Soc. 136, 11043-11049.

Sheldrick, G. M. (2008). Acta Cryst. A64, 112-122. 


\section{data reports}

Sheldrick, G. M. (2015). Acta Cryst. C71, 3-8.

Wang, X., Ng, J. K.-P., Jia, P., Lin, T., Cho, C. M., Xu, J., Lu, X. \& He, C. (2009). Macromolecules, 42, 5534-5544.
Xia, J., Capozzi, B., Wei, S., Strange, M., Batra, A., Moreno, J. R., Amir, E., Amir, R., Solomon, G. C., Venkataraman, L. \& Campos, L. M. (2014). Nano Lett. 14, 2941-2945. 


\title{
supporting information
}

Acta Cryst. (2015). E71, o1099-o1100 [https://doi.org/10.1107/S2056989016000323]

\section{Crystal structure of 1,3-bis\{[4-(acetylsulfanyl) phenyl] ethynyl\}azulene}

\author{
Sebastian Förster, Wilhelm Seichter and Edwin Weber
}

\section{S1. Comment}

Azulene derivatives offer a number of interesting applications especially in the field of molecular electronics (Wang et al., 2009; Puodziukynaite et al., 2014). It ties up with the fact that the non-alternating azulene possesses remarkable electronic and optical properties (Xia et al., 2014). Although the title compound, $\mathrm{C}_{30} \mathrm{H}_{20} \mathrm{O}_{2} \mathrm{~S}_{2}$, (I), is fully conjugated, no flat molecular structure can be observed. Both phenyl rings, fig 1, are rotated out of the plane containing the azulene core [phenyl (C13-C18) $29.0^{\circ}$, phenyl $(\mathrm{C} 23-\mathrm{C} 28) 55.2^{\circ}$ ]. The $\mathrm{C}-\mathrm{S}-\mathrm{C}$ angle of the acetyl protected thiol is slightly smaller compared to that found in an analogous compound,1,3-bis[4-(tert-butylsulfanyl)phenylethynyl]azulene, featuring a tertbutyl protection group at the sulfur atom (Förster et al., 2012). Unlike the previous case, no $\pi \cdots \pi$ interactions are present in the title compound. In all probability, this uncommon phenomenon within the substance class of azulenes (Förster et al., 2014) is related to the non-planar molecular structure and may be caused from packing effects. However, the crystal structure is based on $\mathrm{C}-\mathrm{H} \cdots \mathrm{O}$ hydrogen bonds [ $\left.\mathrm{C} 2-\mathrm{H} 2 \cdots \mathrm{O} 1\left(2.5-\mathrm{x},-0.5+\mathrm{y}, 1.5-\mathrm{z} ; 2.40 \AA, 155.0^{\circ}\right)\right]$ and $\mathrm{C}-\mathrm{H}^{\cdots} \cdots \pi$ interactions [C17- $\left.\mathrm{H} 17 \cdots \mathrm{Cg}(1) 2.69 \AA, 164.9^{\circ} ; \mathrm{C} 20-\mathrm{H} 20 \mathrm{~A} \cdots \mathrm{Cg}(2) 2.89 \AA, 159.0^{\circ}\right]$.

\section{S2. Experimental}

\section{S2.1. Synthesis and crystallization}

The synthesis of the title compound, (I), has already been described (Förster et al. 2012). The crystals were grown from toluene solution by slow evaporation.

\section{S2.2. Refinement}

Crystal data, data collection and structure refinement details are summarized in Table 1. The hydrogen atoms attached to $\mathrm{C}$ were fixed geometrically and treated as riding atoms, with $\mathrm{d}(\mathrm{C}-\mathrm{H})=0.93$ and $\mathrm{Uiso}(\mathrm{H})=1.2 \mathrm{Ueq}(\mathrm{C})$ for aromatic and $\mathrm{Uiso}(\mathrm{H})=1.5 \mathrm{Ueq}(\mathrm{C})$ for methyl groups. 


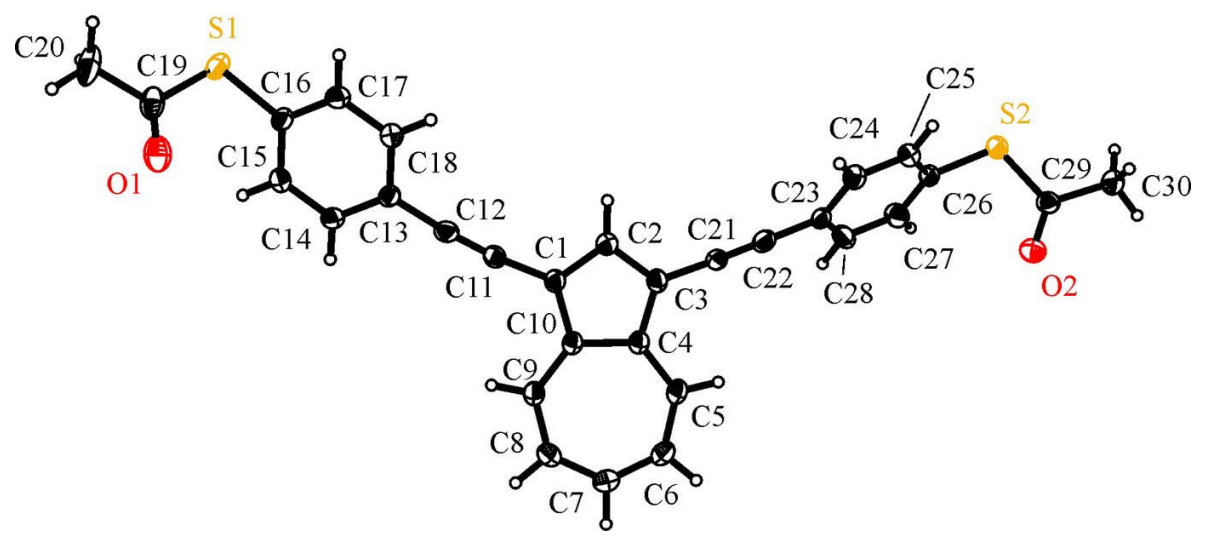

Figure 1

Ellipsoid plot.

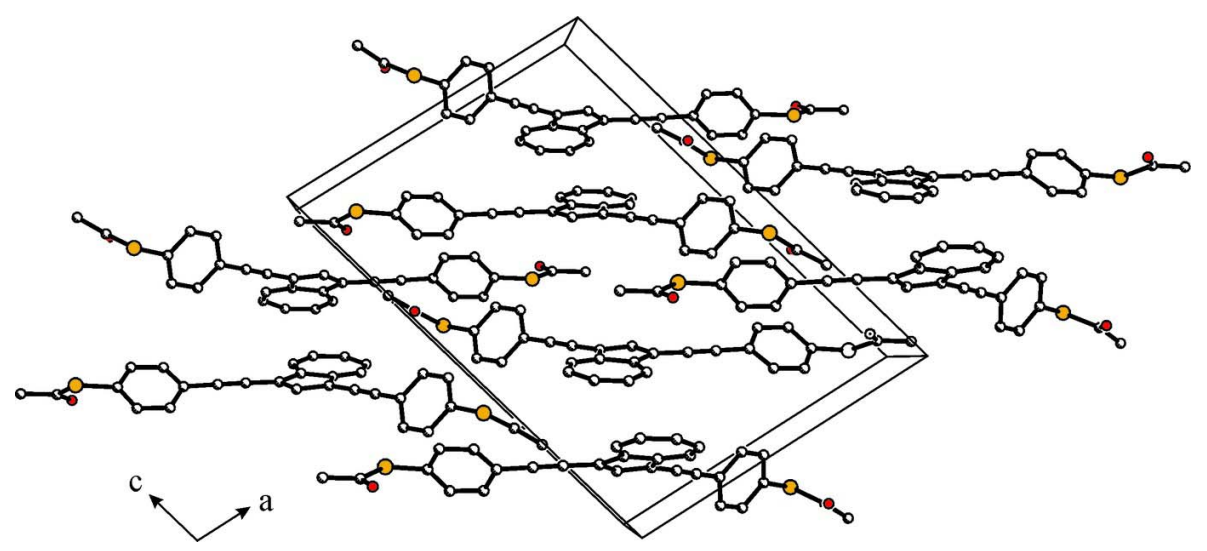

Figure 2

Packing diagram.

1,3-Bis\{[4-(acetylsulfanyl)phenyl] ethynyl\}azulene

Crystal data

$\mathrm{C}_{30} \mathrm{H}_{20} \mathrm{O}_{2} \mathrm{~S}_{2}$

$M_{r}=476.58$

Monoclinic, $P 2_{1} / n$

$a=13.7674$ (3) $\AA$

$b=8.9849(2) \AA$

$c=19.7586(4) \AA$

$\beta=104.022(1)^{\circ}$

$V=2371.28(9) \AA^{3}$

$Z=4$

\section{Data collection}

Bruker Kappa APEX CCD diffractometer

phi and $\omega$ scans

Absorption correction: multi-scan

(SADABS; Bruker, 2008)

$T_{\min }=0.942, T_{\max }=0.963$

36340 measured reflections
$F(000)=992$

$D_{\mathrm{x}}=1.335 \mathrm{Mg} \mathrm{m}^{-3}$

Mo $K \alpha$ radiation, $\lambda=0.71073 \AA$

Cell parameters from 8057 reflections

$\theta=2.5-28.0^{\circ}$

$\mu=0.25 \mathrm{~mm}^{-1}$

$T=100 \mathrm{~K}$

Irregular, green

$0.24 \times 0.23 \times 0.15 \mathrm{~mm}$

5898 independent reflections

4422 reflections with $I>2 \sigma(I)$

$R_{\text {int }}=0.039$

$\theta_{\text {max }}=28.3^{\circ}, \theta_{\min }=2.5^{\circ}$

$h=-18 \rightarrow 18$

$k=-12 \rightarrow 11$

$l=-26 \rightarrow 26$ 


\section{Refinement}

Refinement on $F^{2}$

Least-squares matrix: full

$R\left[F^{2}>2 \sigma\left(F^{2}\right)\right]=0.046$

$w R\left(F^{2}\right)=0.130$

$S=1.03$

5898 reflections

309 parameters

0 restraints
Hydrogen site location: inferred from neighbouring sites

$\mathrm{H}$-atom parameters constrained

$w=1 /\left[\sigma^{2}\left(F_{\mathrm{o}}{ }^{2}\right)+(0.0658 P)^{2}+1.347 P\right]$

where $P=\left(F_{\mathrm{o}}^{2}+2 F_{\mathrm{c}}{ }^{2}\right) / 3$

$(\Delta / \sigma)_{\max }=0.001$

$\Delta \rho_{\max }=0.44$ e $\AA^{-3}$

$\Delta \rho_{\min }=-0.27$ e $\AA^{-3}$

Special details

Geometry. All e.s.d.'s (except the e.s.d. in the dihedral angle between two 1.s. planes) are estimated using the full covariance matrix. The cell e.s.d.'s are taken into account individually in the estimation of e.s.d.'s in distances, angles and torsion angles; correlations between e.s.d.'s in cell parameters are only used when they are defined by crystal symmetry. An approximate (isotropic) treatment of cell e.s.d.'s is used for estimating e.s.d.'s involving 1.s. planes.

Fractional atomic coordinates and isotropic or equivalent isotropic displacement parameters $\left(\AA^{2}\right)$

\begin{tabular}{|c|c|c|c|c|}
\hline & $x$ & $y$ & $z$ & $U_{\text {iso }} * / U_{\text {eq }}$ \\
\hline $\mathrm{S} 1$ & $1.37569(4)$ & $0.11544(6)$ & $0.58441(3)$ & $0.03405(15)$ \\
\hline $\mathrm{S} 2$ & $0.55913(4)$ & $-0.42293(6)$ & $1.10153(3)$ & $0.03144(14)$ \\
\hline $\mathrm{O} 1$ & $1.43557(12)$ & 0.39449 (19) & $0.60090(9)$ & $0.0418(4)$ \\
\hline $\mathrm{O} 2$ & $0.52513(12)$ & $-0.17076(16)$ & $1.16136(8)$ & $0.0361(4)$ \\
\hline $\mathrm{C} 1$ & $0.93413(13)$ & $0.2985(2)$ & $0.81129(9)$ & $0.0229(4)$ \\
\hline $\mathrm{C} 2$ & $0.91025(14)$ & $0.1731(2)$ & $0.84616(10)$ & $0.0248(4)$ \\
\hline $\mathrm{H} 2$ & 0.9425 & 0.0791 & 0.8485 & $0.030 *$ \\
\hline $\mathrm{C} 3$ & $0.83172(14)$ & $0.2075(2)$ & $0.87699(10)$ & $0.0235(4)$ \\
\hline $\mathrm{C} 4$ & $0.80547(13)$ & $0.3585(2)$ & $0.86356(9)$ & $0.0224(4)$ \\
\hline $\mathrm{C} 5$ & $0.73471(14)$ & $0.4346(2)$ & $0.88954(10)$ & 0.0258 \\
\hline H5 & 0.7021 & 0.3782 & 0.9182 & $0.031^{*}$ \\
\hline C6 & $0.70505(15)$ & $0.5828(2)$ & $0.87924(11)$ & $0.0286(4)$ \\
\hline H6 & 0.6574 & 0.6156 & 0.9036 & $0.034 *$ \\
\hline $\mathrm{C} 7$ & $0.73635(14)$ & $0.6887(2)$ & $0.83792(10)$ & $0.0281(4)$ \\
\hline $\mathrm{H} 7$ & 0.7048 & 0.7831 & 0.8366 & $0.034 *$ \\
\hline $\mathrm{C} 8$ & $0.80679(15)$ & $0.6777(2)$ & $0.79820(10)$ & $0.0279(4)$ \\
\hline H8 & 0.8147 & 0.7643 & 0.7725 & $0.033 *$ \\
\hline C9 & $0.86748(14)$ & $0.5575(2)$ & $0.79079(10)$ & $0.0244(4)$ \\
\hline H9 & 0.9129 & 0.5744 & 0.7623 & $0.029 *$ \\
\hline $\mathrm{C} 10$ & $0.86986(13)$ & $0.4173(2)$ & $0.81964(9)$ & $0.0217(4)$ \\
\hline C11 & $1.01077(14)$ & $0.2995(2)$ & $0.77358(10)$ & $0.0235(4)$ \\
\hline C12 & $1.07506(14)$ & $0.2876(2)$ & $0.74377(10)$ & $0.0252(4)$ \\
\hline C13 & $1.15323(13)$ & $0.2606(2)$ & $0.70827(10)$ & $0.0222(4)$ \\
\hline C14 & $1.15694(14)$ & $0.3358(2)$ & $0.64730(10)$ & $0.0261(4)$ \\
\hline H14 & 1.1120 & 0.4155 & 0.6312 & $0.031^{*}$ \\
\hline $\mathrm{C} 15$ & $1.22577(14)$ & $0.2948(2)$ & $0.61000(10)$ & $0.0272(4)$ \\
\hline H15 & 1.2263 & 0.3440 & 0.5675 & $0.033^{*}$ \\
\hline $\mathrm{C} 16$ & $1.29404(13)$ & $0.1821(2)$ & $0.63447(10)$ & $0.0242(4)$ \\
\hline $\mathrm{C} 17$ & $1.29573(14)$ & $0.1142(2)$ & $0.69783(10)$ & 0.0270 \\
\hline H17 & 1.3454 & 0.0420 & 0.7163 & $0.032 *$ \\
\hline
\end{tabular}




$\begin{array}{lllll}\text { C18 } & 1.22522(14) & 0.1517(2) & 0.73400(10) & 0.0269(4) \\ \text { H18 } & 1.2256 & 0.1031 & 0.7768 & 0.032^{*} \\ \text { C19 } & 1.44675(15) & 0.2760(3) & 0.57564(11) & 0.0328(5) \\ \text { C20 } & 1.52157(17) & 0.2459(3) & 0.53316(12) & 0.0483(7) \\ \text { H20A } & 1.4865 & 0.2112 & 0.4866 & 0.072^{*} \\ \text { H20B } & 1.5688 & 0.1692 & 0.5562 & 0.072^{*} \\ \text { H20C } & 1.5582 & 0.3375 & 0.5289 & 0.072^{*} \\ \text { C21 } & 0.78423(15) & 0.1054(2) & 0.91530(9) & 0.0243(4) \\ \text { C22 } & 0.74797(15) & 0.0199(2) & 0.94649(10) & 0.0275(4) \\ \text { C23 } & 0.70236(14) & -0.0862(2) & 0.98362(10) & 0.0246(4) \\ \text { C24 } & 0.76034(15) & -0.1665(2) & 1.03950(10) & 0.0284(4) \\ \text { H24 } & 0.8306 & -0.1504 & 1.0535 & 0.034^{*} \\ \text { C25 } & 0.71595(15) & -0.2691(2) & 1.07442(11) & 0.0289(4) \\ \text { H25 } & 0.7557 & -0.3248 & 1.1119 & 0.035^{*} \\ \text { C26 } & 0.61271(15) & -0.2908(2) & 1.05464(10) & 0.0268(4) \\ \text { C27 } & 0.55486(15) & -0.2111(2) & 1.00026(11) & 0.0318(5) \\ \text { H27 } & 0.4845 & -0.2261 & 0.9870 & 0.038^{*} \\ \text { C28 } & 0.59931(15) & -0.1085(2) & 0.96468(11) & 0.0308(4) \\ \text { H28 } & 0.5592 & -0.0532 & 0.9272 & 0.037^{*} \\ \text { C29 } & 0.52713(14) & -0.3041(2) & 1.16535(10) & 0.0261(4) \\ \text { C30 } & 0.50479(17) & -0.3905(2) & 1.22472(11) & 0.0341(5) \\ \text { H30A } & 0.5636 & -0.3895 & 1.2643 & 0.051^{*} \\ \text { H30B } & 0.4883 & -0.4935 & 1.2100 & 0.051^{*} \\ \text { H30C } & 0.4479 & -0.3451 & 1.2387 & 0.051^{*}\end{array}$

Atomic displacement parameters $\left(\AA^{2}\right)$

\begin{tabular}{lllllll}
\hline & $U^{11}$ & $U^{22}$ & $U^{33}$ & $U^{12}$ & $U^{13}$ & $U^{23}$ \\
\hline S1 & $0.0321(3)$ & $0.0331(3)$ & $0.0435(3)$ & $0.0021(2)$ & $0.0220(2)$ & $-0.0023(2)$ \\
S2 & $0.0425(3)$ & $0.0203(2)$ & $0.0379(3)$ & $-0.0023(2)$ & $0.0221(2)$ & $0.0025(2)$ \\
O1 & $0.0341(8)$ & $0.0477(10)$ & $0.0446(9)$ & $-0.0138(7)$ & $0.0117(7)$ & $-0.0013(8)$ \\
O2 & $0.0478(9)$ & $0.0248(8)$ & $0.0381(8)$ & $0.0010(7)$ & $0.0152(7)$ & $0.0001(6)$ \\
C1 & $0.0234(9)$ & $0.0222(9)$ & $0.0244(9)$ & $-0.0025(7)$ & $0.0085(7)$ & $-0.0004(7)$ \\
C2 & $0.0276(9)$ & $0.0225(9)$ & $0.0260(9)$ & $-0.0003(7)$ & $0.0097(7)$ & $-0.0001(8)$ \\
C3 & $0.0246(9)$ & $0.0238(9)$ & $0.0233(9)$ & $-0.0034(7)$ & $0.0083(7)$ & $0.0004(7)$ \\
C4 & $0.0224(8)$ & $0.0239(9)$ & $0.0217(9)$ & $-0.0023(7)$ & $0.0069(7)$ & $-0.0007(7)$ \\
C5 & $0.0232(9)$ & $0.0301(10)$ & $0.0266(9)$ & $-0.0025(8)$ & $0.0108(7)$ & $-0.0010(8)$ \\
C6 & $0.0259(9)$ & $0.0312(11)$ & $0.0309(10)$ & $0.0015(8)$ & $0.0114(8)$ & $-0.0043(8)$ \\
C7 & $0.0273(9)$ & $0.0266(10)$ & $0.0293(10)$ & $0.0039(8)$ & $0.0048(8)$ & $-0.0027(8)$ \\
C8 & $0.0314(10)$ & $0.0241(10)$ & $0.0277(10)$ & $0.0006(8)$ & $0.0062(8)$ & $0.0041(8)$ \\
C9 & $0.0259(9)$ & $0.0261(10)$ & $0.0224(9)$ & $-0.0024(7)$ & $0.0082(7)$ & $0.0002(7)$ \\
C10 & $0.0208(8)$ & $0.0237(9)$ & $0.0213(8)$ & $-0.0023(7)$ & $0.0067(7)$ & $-0.0012(7)$ \\
C11 & $0.0265(9)$ & $0.0180(9)$ & $0.0269(9)$ & $-0.0045(7)$ & $0.0083(7)$ & $-0.0018(7)$ \\
C12 & $0.0272(9)$ & $0.0179(9)$ & $0.0309(10)$ & $-0.0011(7)$ & $0.0078(8)$ & $-0.0020(7)$ \\
C13 & $0.0222(8)$ & $0.0193(9)$ & $0.0267(9)$ & $-0.0021(7)$ & $0.0093(7)$ & $-0.0034(7)$ \\
C14 & $0.0234(9)$ & $0.0230(10)$ & $0.0331(10)$ & $0.0033(7)$ & $0.0093(8)$ & $0.0033(8)$ \\
C15 & $0.0283(9)$ & $0.0271(10)$ & $0.0284(10)$ & $0.0010(8)$ & $0.0111(8)$ & $0.0059(8)$ \\
C16 & $0.0217(8)$ & $0.0241(10)$ & $0.0293(9)$ & $-0.0010(7)$ & $0.0113(7)$ & $-0.0016(8)$
\end{tabular}




$\begin{array}{lllllll}\text { C17 } & 0.0244(9) & 0.0240(10) & 0.0335(10) & 0.0033(7) & 0.0085(8) & 0.0027(8) \\ \text { C18 } & 0.0293(10) & 0.0256(10) & 0.0279(10) & 0.0009(8) & 0.0108(8) & 0.0023(8) \\ \text { C19 } & 0.0232(9) & 0.0482(14) & 0.0265(10) & -0.0047(9) & 0.0051(8) & 0.0029(9) \\ \text { C20 } & 0.0271(11) & 0.084(2) & 0.0374(12) & -0.0038(12) & 0.0152(9) & 0.0081(13) \\ \text { C21 } & 0.0329(10) & 0.0213(9) & 0.0204(9) & 0.0035(8) & 0.0095(7) & -0.0006(7) \\ \text { C22 } & 0.0301(10) & 0.0246(10) & 0.0304(10) & 0.0002(8) & 0.0126(8) & -0.0050(8) \\ \text { C23 } & 0.0321(10) & 0.0181(9) & 0.0284(9) & -0.0015(7) & 0.0169(8) & -0.0030(7) \\ \text { C24 } & 0.0273(9) & 0.0285(10) & 0.0318(10) & -0.0006(8) & 0.0115(8) & 0.0010(8) \\ \text { C25 } & 0.0325(10) & 0.0262(10) & 0.0304(10) & 0.0028(8) & 0.0124(8) & 0.0046(8) \\ \text { C26 } & 0.0357(10) & 0.0184(9) & 0.0314(10) & -0.0013(8) & 0.0181(8) & 0.0002(8) \\ \text { C27 } & 0.0253(9) & 0.0294(11) & 0.0424(12) & -0.0021(8) & 0.0117(8) & 0.0057(9) \\ \text { C28 } & 0.0309(10) & 0.0271(10) & 0.0358(11) & 0.0019(8) & 0.0110(8) & 0.0096(9) \\ \text { C29 } & 0.0248(9) & 0.0233(10) & 0.0311(10) & -0.0001(7) & 0.0085(8) & 0.0012(8) \\ \text { C30 } & 0.0416(12) & 0.0305(11) & 0.0355(11) & 0.0012(9) & 0.0195(9) & 0.0028(9) \\ \end{array}$

Geometric parameters $\left(\AA,{ }^{\circ}\right)$

\begin{tabular}{|c|c|c|c|}
\hline $\mathrm{S} 1-\mathrm{C} 16$ & $1.7716(19)$ & $\mathrm{C} 14-\mathrm{C} 15$ & $1.384(3)$ \\
\hline $\mathrm{S} 1-\mathrm{C} 19$ & $1.775(2)$ & $\mathrm{C} 14-\mathrm{H} 14$ & 0.9500 \\
\hline $\mathrm{S} 2-\mathrm{C} 26$ & $1.7727(19)$ & $\mathrm{C} 15-\mathrm{C} 16$ & $1.387(3)$ \\
\hline $\mathrm{S} 2-\mathrm{C} 29$ & $1.787(2)$ & $\mathrm{C} 15-\mathrm{H} 15$ & 0.9500 \\
\hline $\mathrm{O} 1-\mathrm{C} 19$ & $1.201(3)$ & $\mathrm{C} 16-\mathrm{C} 17$ & $1.387(3)$ \\
\hline $\mathrm{O} 2-\mathrm{C} 29$ & $1.201(2)$ & $\mathrm{C} 17-\mathrm{C} 18$ & $1.380(3)$ \\
\hline $\mathrm{C} 1-\mathrm{C} 2$ & $1.401(3)$ & C17-H17 & 0.9500 \\
\hline $\mathrm{C} 1-\mathrm{C} 10$ & $1.421(3)$ & $\mathrm{C} 18-\mathrm{H} 18$ & 0.9500 \\
\hline $\mathrm{C} 1-\mathrm{C} 11$ & $1.432(2)$ & $\mathrm{C} 19-\mathrm{C} 20$ & $1.503(3)$ \\
\hline $\mathrm{C} 2-\mathrm{C} 3$ & $1.398(3)$ & $\mathrm{C} 20-\mathrm{H} 20 \mathrm{~A}$ & 0.9800 \\
\hline $\mathrm{C} 2-\mathrm{H} 2$ & 0.9500 & $\mathrm{C} 20-\mathrm{H} 20 \mathrm{~B}$ & 0.9800 \\
\hline $\mathrm{C} 3-\mathrm{C} 4$ & $1.413(3)$ & $\mathrm{C} 20-\mathrm{H} 20 \mathrm{C}$ & 0.9800 \\
\hline $\mathrm{C} 3-\mathrm{C} 21$ & $1.443(3)$ & $\mathrm{C} 21-\mathrm{C} 22$ & $1.170(3)$ \\
\hline $\mathrm{C} 4-\mathrm{C} 5$ & $1.386(3)$ & $\mathrm{C} 22-\mathrm{C} 23$ & $1.437(3)$ \\
\hline $\mathrm{C} 4-\mathrm{C} 10$ & $1.480(2)$ & $\mathrm{C} 23-\mathrm{C} 28$ & $1.391(3)$ \\
\hline $\mathrm{C} 5-\mathrm{C} 6$ & $1.393(3)$ & $\mathrm{C} 23-\mathrm{C} 24$ & $1.396(3)$ \\
\hline $\mathrm{C} 5-\mathrm{H} 5$ & 0.9500 & $\mathrm{C} 24-\mathrm{C} 25$ & $1.380(3)$ \\
\hline $\mathrm{C} 6-\mathrm{C} 7$ & $1.388(3)$ & $\mathrm{C} 24-\mathrm{H} 24$ & 0.9500 \\
\hline $\mathrm{C} 6-\mathrm{H} 6$ & 0.9500 & $\mathrm{C} 25-\mathrm{C} 26$ & $1.394(3)$ \\
\hline $\mathrm{C} 7-\mathrm{C} 8$ & $1.391(3)$ & $\mathrm{C} 25-\mathrm{H} 25$ & 0.9500 \\
\hline $\mathrm{C} 7-\mathrm{H} 7$ & 0.9500 & $\mathrm{C} 26-\mathrm{C} 27$ & $1.374(3)$ \\
\hline $\mathrm{C} 8-\mathrm{C} 9$ & $1.394(3)$ & $\mathrm{C} 27-\mathrm{C} 28$ & $1.388(3)$ \\
\hline $\mathrm{C} 8-\mathrm{H} 8$ & 0.9500 & $\mathrm{C} 27-\mathrm{H} 27$ & 0.9500 \\
\hline $\mathrm{C} 9-\mathrm{C} 10$ & $1.380(3)$ & $\mathrm{C} 28-\mathrm{H} 28$ & 0.9500 \\
\hline $\mathrm{C} 9-\mathrm{H} 9$ & 0.9500 & $\mathrm{C} 29-\mathrm{C} 30$ & $1.500(3)$ \\
\hline $\mathrm{C} 11-\mathrm{C} 12$ & $1.181(3)$ & $\mathrm{C} 30-\mathrm{H} 30 \mathrm{~A}$ & 0.9800 \\
\hline $\mathrm{C} 12-\mathrm{C} 13$ & $1.440(3)$ & $\mathrm{C} 30-\mathrm{H} 30 \mathrm{~B}$ & 0.9800 \\
\hline $\mathrm{C} 13-\mathrm{C} 14$ & $1.393(3)$ & $\mathrm{C} 30-\mathrm{H} 30 \mathrm{C}$ & 0.9800 \\
\hline $\mathrm{C} 13-\mathrm{C} 18$ & $1.398(3)$ & & \\
\hline $\mathrm{C} 16-\mathrm{S} 1-\mathrm{C} 19$ & $102.66(10)$ & $\mathrm{C} 17-\mathrm{C} 16-\mathrm{S} 1$ & $118.76(15)$ \\
\hline
\end{tabular}




\begin{tabular}{|c|c|c|c|}
\hline $\mathrm{C} 26-\mathrm{S} 2-\mathrm{C} 29$ & $100.01(9)$ & $\mathrm{C} 18-\mathrm{C} 17-\mathrm{C} 16$ & $120.01(18)$ \\
\hline $\mathrm{C} 2-\mathrm{C} 1-\mathrm{C} 10$ & $108.67(16)$ & $\mathrm{C} 18-\mathrm{C} 17-\mathrm{H} 17$ & 120.0 \\
\hline $\mathrm{C} 2-\mathrm{C} 1-\mathrm{C} 11$ & $123.48(17)$ & $\mathrm{C} 16-\mathrm{C} 17-\mathrm{H} 17$ & 120.0 \\
\hline $\mathrm{C} 10-\mathrm{C} 1-\mathrm{C} 11$ & $127.85(17)$ & $\mathrm{C} 17-\mathrm{C} 18-\mathrm{C} 13$ & $120.56(18)$ \\
\hline $\mathrm{C} 3-\mathrm{C} 2-\mathrm{C} 1$ & $109.57(17)$ & $\mathrm{C} 17-\mathrm{C} 18-\mathrm{H} 18$ & 119.7 \\
\hline $\mathrm{C} 3-\mathrm{C} 2-\mathrm{H} 2$ & 125.2 & $\mathrm{C} 13-\mathrm{C} 18-\mathrm{H} 18$ & 119.7 \\
\hline $\mathrm{C} 1-\mathrm{C} 2-\mathrm{H} 2$ & 125.2 & $\mathrm{O} 1-\mathrm{C} 19-\mathrm{C} 20$ & $124.5(2)$ \\
\hline $\mathrm{C} 2-\mathrm{C} 3-\mathrm{C} 4$ & $108.68(16)$ & $\mathrm{O} 1-\mathrm{C} 19-\mathrm{S} 1$ & $123.40(16)$ \\
\hline $\mathrm{C} 2-\mathrm{C} 3-\mathrm{C} 21$ & $125.77(18)$ & $\mathrm{C} 20-\mathrm{C} 19-\mathrm{S} 1$ & $112.13(18)$ \\
\hline $\mathrm{C} 4-\mathrm{C} 3-\mathrm{C} 21$ & $125.55(17)$ & $\mathrm{C} 19-\mathrm{C} 20-\mathrm{H} 20 \mathrm{~A}$ & 109.5 \\
\hline $\mathrm{C} 5-\mathrm{C} 4-\mathrm{C} 3$ & $125.09(17)$ & $\mathrm{C} 19-\mathrm{C} 20-\mathrm{H} 20 \mathrm{~B}$ & 109.5 \\
\hline $\mathrm{C} 5-\mathrm{C} 4-\mathrm{C} 10$ & $128.00(18)$ & $\mathrm{H} 20 \mathrm{~A}-\mathrm{C} 20-\mathrm{H} 20 \mathrm{~B}$ & 109.5 \\
\hline $\mathrm{C} 3-\mathrm{C} 4-\mathrm{C} 10$ & $106.88(16)$ & $\mathrm{C} 19-\mathrm{C} 20-\mathrm{H} 20 \mathrm{C}$ & 109.5 \\
\hline $\mathrm{C} 4-\mathrm{C} 5-\mathrm{C} 6$ & $128.65(18)$ & $\mathrm{H} 20 \mathrm{~A}-\mathrm{C} 20-\mathrm{H} 20 \mathrm{C}$ & 109.5 \\
\hline $\mathrm{C} 4-\mathrm{C} 5-\mathrm{H} 5$ & 115.7 & $\mathrm{H} 20 \mathrm{~B}-\mathrm{C} 20-\mathrm{H} 20 \mathrm{C}$ & 109.5 \\
\hline $\mathrm{C} 6-\mathrm{C} 5-\mathrm{H} 5$ & 115.7 & $\mathrm{C} 22-\mathrm{C} 21-\mathrm{C} 3$ & $178.1(2)$ \\
\hline $\mathrm{C} 7-\mathrm{C} 6-\mathrm{C} 5$ & $128.24(18)$ & $\mathrm{C} 21-\mathrm{C} 22-\mathrm{C} 23$ & $178.9(2)$ \\
\hline $\mathrm{C} 7-\mathrm{C} 6-\mathrm{H} 6$ & 115.9 & $\mathrm{C} 28-\mathrm{C} 23-\mathrm{C} 24$ & $119.25(17)$ \\
\hline $\mathrm{C} 5-\mathrm{C} 6-\mathrm{H} 6$ & 115.9 & $\mathrm{C} 28-\mathrm{C} 23-\mathrm{C} 22$ & $120.10(18)$ \\
\hline $\mathrm{C} 6-\mathrm{C} 7-\mathrm{C} 8$ & $129.88(19)$ & $\mathrm{C} 24-\mathrm{C} 23-\mathrm{C} 22$ & $120.64(18)$ \\
\hline $\mathrm{C} 6-\mathrm{C} 7-\mathrm{H} 7$ & 115.1 & $\mathrm{C} 25-\mathrm{C} 24-\mathrm{C} 23$ & $120.20(18)$ \\
\hline $\mathrm{C} 8-\mathrm{C} 7-\mathrm{H} 7$ & 115.1 & $\mathrm{C} 25-\mathrm{C} 24-\mathrm{H} 24$ & 119.9 \\
\hline $\mathrm{C} 7-\mathrm{C} 8-\mathrm{C} 9$ & $129.18(19)$ & $\mathrm{C} 23-\mathrm{C} 24-\mathrm{H} 24$ & 119.9 \\
\hline $\mathrm{C} 7-\mathrm{C} 8-\mathrm{H} 8$ & 115.4 & $\mathrm{C} 24-\mathrm{C} 25-\mathrm{C} 26$ & $119.92(19)$ \\
\hline $\mathrm{C} 9-\mathrm{C} 8-\mathrm{H} 8$ & 115.4 & $\mathrm{C} 24-\mathrm{C} 25-\mathrm{H} 25$ & 120.0 \\
\hline $\mathrm{C} 10-\mathrm{C} 9-\mathrm{C} 8$ & $128.20(18)$ & $\mathrm{C} 26-\mathrm{C} 25-\mathrm{H} 25$ & 120.0 \\
\hline $\mathrm{C} 10-\mathrm{C} 9-\mathrm{H} 9$ & 115.9 & $\mathrm{C} 27-\mathrm{C} 26-\mathrm{C} 25$ & $120.30(18)$ \\
\hline $\mathrm{C} 8-\mathrm{C} 9-\mathrm{H} 9$ & 115.9 & $\mathrm{C} 27-\mathrm{C} 26-\mathrm{S} 2$ & $121.48(15)$ \\
\hline $\mathrm{C} 9-\mathrm{C} 10-\mathrm{C} 1$ & $126.22(17)$ & $\mathrm{C} 25-\mathrm{C} 26-\mathrm{S} 2$ & $118.21(15)$ \\
\hline $\mathrm{C} 9-\mathrm{C} 10-\mathrm{C} 4$ & $127.60(17)$ & $\mathrm{C} 26-\mathrm{C} 27-\mathrm{C} 28$ & $119.96(19)$ \\
\hline $\mathrm{C} 1-\mathrm{C} 10-\mathrm{C} 4$ & $106.17(16)$ & $\mathrm{C} 26-\mathrm{C} 27-\mathrm{H} 27$ & 120.0 \\
\hline $\mathrm{C} 12-\mathrm{C} 11-\mathrm{C} 1$ & $174.3(2)$ & $\mathrm{C} 28-\mathrm{C} 27-\mathrm{H} 27$ & 120.0 \\
\hline $\mathrm{C} 11-\mathrm{C} 12-\mathrm{C} 13$ & $175.5(2)$ & $\mathrm{C} 27-\mathrm{C} 28-\mathrm{C} 23$ & $120.36(19)$ \\
\hline $\mathrm{C} 14-\mathrm{C} 13-\mathrm{C} 18$ & $118.85(17)$ & $\mathrm{C} 27-\mathrm{C} 28-\mathrm{H} 28$ & 119.8 \\
\hline $\mathrm{C} 14-\mathrm{C} 13-\mathrm{C} 12$ & $121.86(17)$ & $\mathrm{C} 23-\mathrm{C} 28-\mathrm{H} 28$ & 119.8 \\
\hline $\mathrm{C} 18-\mathrm{C} 13-\mathrm{C} 12$ & $119.23(17)$ & $\mathrm{O} 2-\mathrm{C} 29-\mathrm{C} 30$ & $124.21(19)$ \\
\hline $\mathrm{C} 15-\mathrm{C} 14-\mathrm{C} 13$ & $120.34(18)$ & $\mathrm{O} 2-\mathrm{C} 29-\mathrm{S} 2$ & $123.75(16)$ \\
\hline $\mathrm{C} 15-\mathrm{C} 14-\mathrm{H} 14$ & 119.8 & $\mathrm{C} 30-\mathrm{C} 29-\mathrm{S} 2$ & $112.03(14)$ \\
\hline C13-C14-H14 & 119.8 & $\mathrm{C} 29-\mathrm{C} 30-\mathrm{H} 30 \mathrm{~A}$ & 109.5 \\
\hline $\mathrm{C} 14-\mathrm{C} 15-\mathrm{C} 16$ & $120.16(17)$ & $\mathrm{C} 29-\mathrm{C} 30-\mathrm{H} 30 \mathrm{~B}$ & 109.5 \\
\hline $\mathrm{C} 14-\mathrm{C} 15-\mathrm{H} 15$ & 119.9 & $\mathrm{H} 30 \mathrm{~A}-\mathrm{C} 30-\mathrm{H} 30 \mathrm{~B}$ & 109.5 \\
\hline $\mathrm{C} 16-\mathrm{C} 15-\mathrm{H} 15$ & 119.9 & $\mathrm{C} 29-\mathrm{C} 30-\mathrm{H} 30 \mathrm{C}$ & 109.5 \\
\hline $\mathrm{C} 15-\mathrm{C} 16-\mathrm{C} 17$ & $119.82(17)$ & $\mathrm{H} 30 \mathrm{~A}-\mathrm{C} 30-\mathrm{H} 30 \mathrm{C}$ & 109.5 \\
\hline $\mathrm{C} 15-\mathrm{C} 16-\mathrm{S} 1$ & $121.35(15)$ & $\mathrm{H} 30 \mathrm{~B}-\mathrm{C} 30-\mathrm{H} 30 \mathrm{C}$ & 109.5 \\
\hline $\mathrm{C} 10-\mathrm{C} 1-\mathrm{C} 2-\mathrm{C} 3$ & $0.1(2)$ & $\mathrm{C} 13-\mathrm{C} 14-\mathrm{C} 15-\mathrm{C} 16$ & $-2.4(3)$ \\
\hline $\mathrm{C} 11-\mathrm{C} 1-\mathrm{C} 2-\mathrm{C} 3$ & $-179.26(17)$ & $\mathrm{C} 14-\mathrm{C} 15-\mathrm{C} 16-\mathrm{C} 17$ & $-2.5(3)$ \\
\hline $\mathrm{C} 1-\mathrm{C} 2-\mathrm{C} 3-\mathrm{C} 4$ & $-1.4(2)$ & $\mathrm{C} 14-\mathrm{C} 15-\mathrm{C} 16-\mathrm{S} 1$ & $174.31(15)$ \\
\hline
\end{tabular}




$\begin{array}{llll}\mathrm{C} 1-\mathrm{C} 2-\mathrm{C} 3-\mathrm{C} 21 & 177.71(18) & \mathrm{C} 19-\mathrm{S} 1-\mathrm{C} 16-\mathrm{C} 15 & 61.42(18) \\ \mathrm{C} 2-\mathrm{C} 3-\mathrm{C} 4-\mathrm{C} 5 & -175.89(18) & \mathrm{C} 19-\mathrm{S} 1-\mathrm{C} 16-\mathrm{C} 17 & -121.78(17) \\ \mathrm{C} 21-\mathrm{C} 3-\mathrm{C} 4-\mathrm{C} 5 & 5.0(3) & \mathrm{C} 15-\mathrm{C} 16-\mathrm{C} 17-\mathrm{C} 18 & 4.4(3) \\ \mathrm{C} 2-\mathrm{C} 3-\mathrm{C} 4-\mathrm{C} 10 & 2.2(2) & \mathrm{S} 1-\mathrm{C} 16-\mathrm{C} 17-\mathrm{C} 18 & -172.40(15) \\ \mathrm{C} 21-\mathrm{C} 3-\mathrm{C} 4-\mathrm{C} 10 & -176.99(17) & \mathrm{C} 16-\mathrm{C} 17-\mathrm{C} 18-\mathrm{C} 13 & -1.6(3) \\ \mathrm{C} 3-\mathrm{C} 4-\mathrm{C} 5-\mathrm{C} 6 & 179.6(2) & \mathrm{C} 14-\mathrm{C} 13-\mathrm{C} 18-\mathrm{C} 17 & -3.1(3) \\ \mathrm{C} 10-\mathrm{C} 4-\mathrm{C} 5-\mathrm{C} 6 & 1.9(3) & \mathrm{C} 12-\mathrm{C} 13-\mathrm{C} 18-\mathrm{C} 17 & 174.31(18) \\ \mathrm{C} 4-\mathrm{C} 5-\mathrm{C} 6-\mathrm{C} 7 & 3.0(4) & \mathrm{C} 16-\mathrm{S} 1-\mathrm{C} 19-\mathrm{O} 1 & 0.8(2) \\ \mathrm{C} 5-\mathrm{C} 6-\mathrm{C} 7-\mathrm{C} 8 & -2.4(4) & \mathrm{C} 16-\mathrm{S} 1-\mathrm{C} 19-\mathrm{C} 20 & -179.13(15) \\ \mathrm{C} 6-\mathrm{C} 7-\mathrm{C} 8-\mathrm{C} 9 & -2.1(4) & \mathrm{C} 28-\mathrm{C} 23-\mathrm{C} 24-\mathrm{C} 25 & 1.4(3) \\ \mathrm{C} 7-\mathrm{C} 8-\mathrm{C} 9-\mathrm{C} 10 & 2.4(4) & \mathrm{C} 22-\mathrm{C} 23-\mathrm{C} 24-\mathrm{C} 25 & -179.43(18) \\ \mathrm{C} 8-\mathrm{C} 9-\mathrm{C} 10-\mathrm{C} 1 & -179.16(19) & \mathrm{C} 23-\mathrm{C} 24-\mathrm{C} 25-\mathrm{C} 26 & -1.1(3) \\ \mathrm{C} 8-\mathrm{C} 9-\mathrm{C} 10-\mathrm{C} 4 & 2.5(3) & \mathrm{C} 24-\mathrm{C} 25-\mathrm{C} 26-\mathrm{C} 27 & 0.3(3) \\ \mathrm{C} 2-\mathrm{C} 1-\mathrm{C} 10-\mathrm{C} 9 & -177.39(18) & \mathrm{C} 24-\mathrm{C} 25-\mathrm{C} 26-\mathrm{S} 2 & -179.55(15) \\ \mathrm{C} 11-\mathrm{C} 1-\mathrm{C} 10-\mathrm{C} 9 & 1.9(3) & \mathrm{C} 29-\mathrm{S} 2-\mathrm{C} 26-\mathrm{C} 27 & -86.86(18) \\ \mathrm{C} 2-\mathrm{C} 1-\mathrm{C} 10-\mathrm{C} 4 & 1.2(2) & \mathrm{C} 29-\mathrm{S} 2-\mathrm{C} 26-\mathrm{C} 25 & 92.98(17) \\ \mathrm{C} 11-\mathrm{C} 1-\mathrm{C} 10-\mathrm{C} 4 & -179.46(18) & \mathrm{C} 25-\mathrm{C} 26-\mathrm{C} 27-\mathrm{C} 28 & 0.1(3) \\ \mathrm{C} 5-\mathrm{C} 4-\mathrm{C} 10-\mathrm{C} 9 & -5.5(3) & \mathrm{S} 2-\mathrm{C} 26-\mathrm{C} 27-\mathrm{C} 28 & 179.98(16) \\ \mathrm{C} 3-\mathrm{C} 4-\mathrm{C} 10-\mathrm{C} 9 & 176.53(18) & \mathrm{C} 26-\mathrm{C} 27-\mathrm{C} 28-\mathrm{C} 23 & 0.2(3) \\ \mathrm{C} 5-\mathrm{C} 4-\mathrm{C} 10-\mathrm{C} 1 & 175.89(19) & \mathrm{C} 24-\mathrm{C} 23-\mathrm{C} 28-\mathrm{C} 27 & -1.0(3) \\ \mathrm{C} 3-\mathrm{C} 4-\mathrm{C} 10-\mathrm{C} 1 & -2.1(2) & \mathrm{C} 22-\mathrm{C} 23-\mathrm{C} 28-\mathrm{C} 27 & 179.86(19) \\ \mathrm{C} 18-\mathrm{C} 13-\mathrm{C} 14-\mathrm{C} 15 & 5.1(3) & \mathrm{C} 26-\mathrm{S} 2-\mathrm{C} 29-\mathrm{O} 2 & 14.4(2) \\ \mathrm{C} 12-\mathrm{C} 13-\mathrm{C} 14-\mathrm{C} 15 & -172.25(18) & \mathrm{C} 26-\mathrm{S} 2-\mathrm{C} 29-\mathrm{C} 30 & -164.72(15) \\ & & & \end{array}$

Hydrogen-bond geometry $\left(A,{ }^{\circ}\right)$

$\mathrm{Cg} 1$ is the mid-point of $\mathrm{C} 11-\mathrm{C} 12$ and $\mathrm{Cg} 2$ is the centroid of the $\mathrm{C} 1-\mathrm{C} 4 / \mathrm{C} 10$ ring.

\begin{tabular}{lllll}
\hline$D-\mathrm{H} \cdots A$ & $D-\mathrm{H}$ & $\mathrm{H} \cdots A$ & $D \cdots A$ & $D-\mathrm{H} \cdots A$ \\
\hline $\mathrm{C} 2-\mathrm{H} 2 \cdots \mathrm{O} 1^{\mathrm{i}}$ & 0.95 & 2.40 & $3.285(3)$ & 155 \\
$\mathrm{C} 17-\mathrm{H} 1 \cdots C g 1$ & 0.95 & 2.69 & $3.612(3)$ & 165 \\
$\mathrm{C} 20-\mathrm{H} 20 A \cdots C g 2^{\mathrm{ii}}$ & 0.98 & 2.89 & $3.835(2)$ & 162 \\
\hline
\end{tabular}

Symmetry codes: (i) $-x+5 / 2, y-1 / 2,-z+3 / 2$; (ii) $x+1 / 2,-y+1 / 2, z-1 / 2$. 